\title{
Quantitative effects of rapid heating on soot-particle sizing through analysis of two-pulse LII
}

\author{
E. Cenker ${ }^{1}(\bowtie)$ and W.L. Roberts ${ }^{1}$
}

${ }^{1}$ King Abdullah University of Science and Technology (KAUST), Clean Combustion Research Center (CCRC), Thuwal 23955-6900, Saudi Arabia

Corresponding author:

emre.cenker@kaust.edu.sa

\begin{abstract}
During the rapid laser pulse heating and consecutive cooling in laser-induced incandescence (LII), soot particles may undergo thermal annealing and sublimation processes which leads to a permanent change in its optical properties and its primary particle size, respectively. Overall effects of these two processes on soot and LII modelbased particle sizing are investigated by measuring the two-color time-resolved (2C-TiRe) LII signal decay from inflame soot after two consecutive laser pulses at 1064-nm wavelength. Experiments are carried out on a nonpremixed laminar ethylene/air flame from a Santoro burner with both low and moderate laser fluences suitable for particle sizing. The probe volume is set to a radial position close to the flame axis where the soot particles are known to be immature or less graphitic. With the first pulse, soot is preheated, and the LII signal after the consecutive second pulse is used for analysis. The two-color incandescence emission technique is used for the pyrometric determination of the LII-heated peak soot temperature at the second pulse. A new LII simulation tool is developed which accounts for particle heating via absorption and annealing, and cooling via sublimation, conduction, and radiation with various existing submodels from the literature. The same approach of using two laser pulses is implemented in the simulations. Measurements indicate that thermal annealing and associated absorption enhancement becomes important at laser fluences above $0.17 \mathrm{~J} / \mathrm{cm}^{2}$ for the immature in-flame soot. After a heating pulse at $0.33 \mathrm{~J} / \mathrm{cm}^{2}$, the increase of the soot absorption function is calculated as $35 \%$ by using the temperature measured at the second pulse and an absorption model based on the Rayleigh approximation. Present annealing model, on the other hand, predicts graphitization of soot even in the absence of laser heating at typical flame temperatures. Recorded experimental LII signal decays and LII-heated peak soot temperature information are used for particle sizing with the LII modeling to assess the effects of sublimation. A reduction in
\end{abstract}


particle size due to sublimation starts at a laser fluence of $0.1 \mathrm{~J} / \mathrm{cm}^{2}$ for the in-flame soot. After a heating pulse at $0.33 \mathrm{~J} / \mathrm{cm}^{2}$, the particle loses $55 \%$ of its initial mass.

\section{Keywords}

Soot particle size, LII modeling, sublimation, and thermal annealing 


\section{Introduction}

Time-resolved laser-induced incandescence (TiRe-LII) is an optical in situ technique for measuring the soot particle size [1]. Soot particles are heated via absorption of light from a laser pulse and the subsequent blackbody radiation is recorded during the cooling phase. Quantitative particle-size information can be obtained from a best-fit comparison of the temporal signal decay and simulations based on the particle's energy- and mass-balance equations [2]. The energy absorbed by the particles and resulting high temperatures, however, may distort the particles permanently from their initial state and such changes should also be considered in simulations to describe the behavior of particles and LII process accurately.

The pioneering works of Vander Wal et al. [3-5] have shown transmission electron microscopy (TEM) images of laser-heated soot extracted from a flame. More recently, De luliis et al. [6] and Michelsen et al. [7] performed similar analyses with cold soot sampled from quenched flames and Bambha et al. [8] repeated the same analysis with coated particles. In all these works, the effects of different heating levels on soot were reported by varying the laser fluence prior to TEM sampling. Soot particles undergo a thermal annealing process due to the rapid heating and consecutive cooling which leads to a permanent transformation of the soot properties. At a fluence of $0.3 \mathrm{~J} / \mathrm{cm}^{2}$ with $1064-\mathrm{nm}$ excitation, the soot particle perimeter remains unchanged, while the primary particle's internal structure becomes irreversibly modified. With further increases in the fluence level, ribbons of several carbon layers appeared at the perimeter of the particles [9]. At fluences below $0.2 \mathrm{~J} / \mathrm{cm}^{2}$, on the other hand, noticeable fragmentation or coalescence of the aggregate structure were not observed with the TEM analysis.

For a better understanding of effects of rapid heating on soot, Vander Wal et al. [4,9] performed two pulse LII experiments where in-flame soot is preheated with the first pulse, and the LII signal from the consecutive second pulse is measured. By analyzing the ratio of the time-gated peak LII signal from each of the pulses, they observed that for modest heating with the first laser pulse $\left(\sim 0.1 \mathrm{~J} / \mathrm{cm}^{2}\right)$, the signal induced by the second pulse is enhanced, indicating that annealing and graphitization could be leading to enhanced absorption and emission characteristics of the soot. For higher laser fluences (up to $0.45 \mathrm{~J} / \mathrm{cm}^{2}$ ), they reported that the induced morphological changes resulted in a decrease in the absorption and emission properties of the soot. At further elevated fluences, they determined that interior and exterior mass loss occurs through sublimation. However, optical properties or particle temperature during the LII process as required for validation of LII modeling were not explicitly measured, with arguments based only on the LII peak intensity and decay curves.

In a recent study [10], Saffaripour et al. investigated the variations in the optical properties of in-flame soot due to rapid laser heating by measuring the time-resolved extinction coefficient of soot. They reported that the rapid 
enhancement of absorption occurs at the time of laser heating, suggesting an increase in soot emission propensity. They also showed evidence for significant loss of soot mass at fluences as low as $0.103 \mathrm{~J} / \mathrm{cm}^{2}$ in a non-premixed flame, consistent with the predictions of LII models.

The annealing process has been accounted for in a LII model for the first time by Michelsen [11]. This is the only current known annealing model in the LII community, and it addresses structural changes in the carbonaceous particles by including phase-dependent properties. Specifically, annealing of the particle in the model is a function of the temperature, and it describes an increase in the mass fraction of graphitized soot. Referring to previous studies on soot and graphite in the literature, Michelsen used a lower soot absorption function for the soot particle once it is annealed. With the given parameter set in the original work of Michelsen [11], the mass fraction conversion of the soot to graphite takes place even at flame temperatures. This eventually leads to an absolute decrease in the absorption and emission properties of the soot for all laser-heating scenarios. This appears to contradict the experimental measurements of soot annealing effects shown by Vander Wal et al. [4,9]. In a recent work [12], Michelsen's group reported a modified expression for soot absorption function which also predicts an enhancement of the light absorption rate after the graphitization. A modification in the mass fraction conversion rate, however, was not mentioned.

In this present work, the overall effects of these annealing and sublimation processes on particle sizing with LII modeling are investigated quantitatively by measuring the two-color time-resolved (2C-TiRe) LII signal decay from in-flame soot for low and moderate laser fluences. Similar to the works of Vander Wal et al. [4,9], optical measurements are performed using a two-pulse LII system. In addition, LII-heated peak soot temperature is measured with two-color pyrometry. The experimental work is coupled with simulations of the LII signal. A new LII simulation tool is developed which accounts for particle heating via absorption of laser light and annealing and cooling via sublimation, conduction, and radiation. An approach using multiple laser pulses is implemented in the simulations. The response of the LII signal and the particle temperature under different two-pulse heating scenarios are analyzed. The measured LII-heated peak soot temperature, named as heat-up temperature hereafter, is used to set the initial conditions for the model.

\section{Experimental setup}

Particle-size measurements were performed in a non-premixed laminar ethylene/air flame from a Santoro burner equipped with a chimney [13]. The burner was operated under standard conditions $\left(\mathrm{C}_{2} \mathrm{H}_{4}: 0.232\right.$ standard liters per minute (s/m), air co-flow: $43 \mathrm{slm}$ at $293 \mathrm{~K}$ and $1 \mathrm{bar})$. The visible flame height was $9.8 \mathrm{~cm}$. A schematic of the experiment is shown in Fig. 1. 


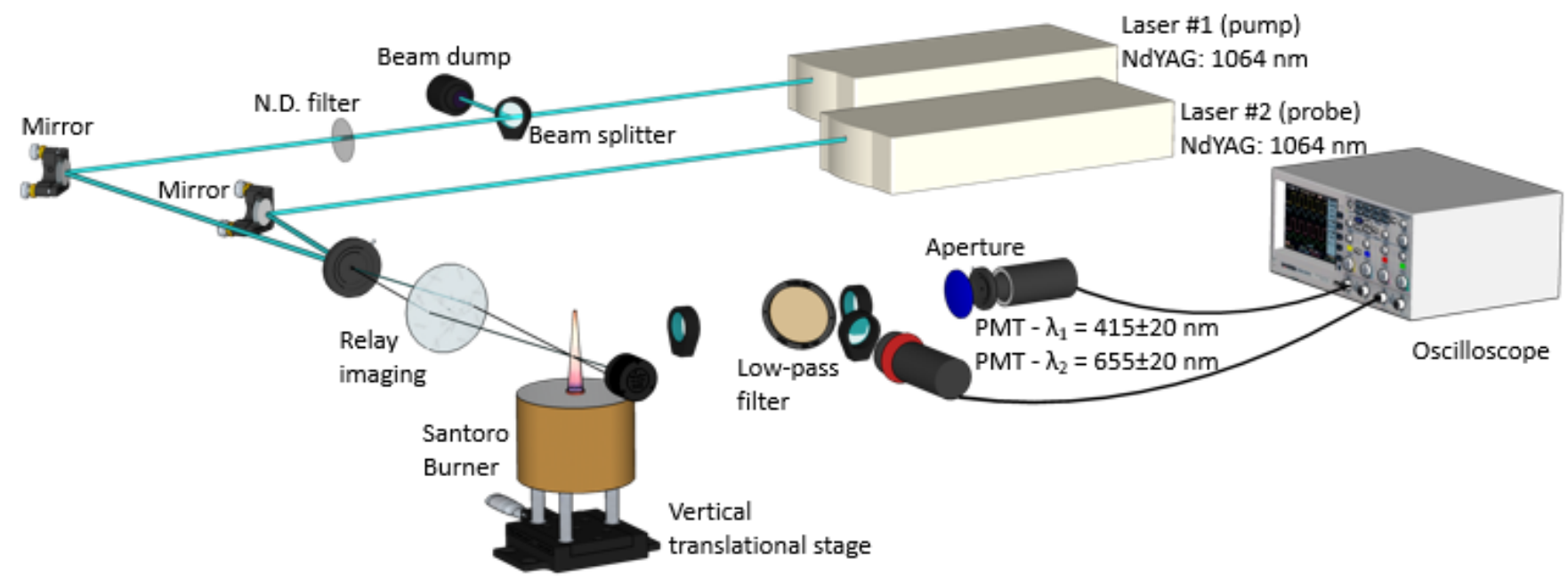

Fig. 1 Experimental arrangement

Two Nd:YAG lasers (SpectraPhysics Pro 250-10 and Continuum SL III-10) were operated at their fundamental output wavelength of 1064-nm. The lasers were triggered with a delay generator. In both lasers, the energy was controlled through a combination of beam splitters, absorptive-type neutral density (N.D.) filters and changing the Q-switch delay. The two laser beams were set at an oblique angle with two dichroic mirrors resulting in an overlapping of both beams on a 1-mm circular aperture. The distance between the mirrors and the aperture is longer than two meters and the oblique angle was less than one degree (exaggerated in the schematic for clarity). The aperture crops both beams into a circular shape that is relay-imaged into the center of flame with a spherical lens at a $2 f$ distance. The relay lens with a $20 \mathrm{~cm}$ focal length also provides a slow convergence of the two oblique beams at the flame location. The complete overlapping of the two beams inside the flame was verified with photosensitive paper. The laser beams were relayed $\sim 1 \mathrm{~mm}$ off the burner axis on the detection unit side at a height of $5 \mathrm{~cm}$ above the burner. This radial location has a slightly higher soot mass with respect to the burner axis location, thus better signal-to-noise ratios can be achieved. Furthermore, smaller soot mass between the probe volume and the detection units reduces the attenuation of LII signal, i.e. signal trapping, which may cause a systematic error in pyrometric determination of temperature due to wavelength-dependent extinction of soot. More importantly, an ex-situ characterization of aggregate and particle size was previously performed at the same radial location [14] and the complete TEM dataset provides reliable information of number of primary particles in an aggregate to be used in LII modeling.

The LII signal was detected with two fast photomultipliers (Hamamatsu R9880U-210 and -20) coupled with bandpass filters (Semrock, $435 \pm 20$ and $655 \pm 20 \mathrm{~nm}$ ) and stored on a $2.5 \mathrm{GHz}$ oscilloscope. The photomultipliers were combined with a one-to-one imaging system of spherical lenses, circular apertures and a longpass filter (496 
$\mathrm{nm}$ ) as shown in Fig. 1. The collection volume (circular probe zone) in the flame has a diameter of less than $1 \mathrm{~mm}$. The variation of thermodynamic properties of soot within the collection volume is believed to be marginal [15]. The temporal alignment of acquired signal at two colors was verified via exciting a laser viewing card (Thorlabs VRC2) placed at the burner axis - bandpass filters were not installed during this measurement.

For two-color pyrometry, spectral bands were selected according to the recommendation of [16]. The relative sensitivity of both detectors was calibrated against the well-documented temperature of the standard Santoro flame at a height of $5 \mathrm{~cm}$ above the burner, as shown in ref. [17]. In this present work, however, the methodology of defining the reference flame temperature has been modified. Recorded signals are inherently line-of-sight integrated flame luminosity along the radial direction. The resulting color ratio is strongly biased to information at the flame edge where the soot temperature is maximum. This is due to the fact that the radiation power is proportional to the fourth power of the temperature. To calculate the line-of-sight averaged flame temperature from the line-of-sight integrated incandescence signal, the bath-gas temperature distribution of the flame and the soot volume fraction distribution were imported from the literature $[15,18]$ (see Fig. 2). These temperature and soot volume fraction information were divided in $0.1 \mathrm{~mm}$ thick layers along the radial direction. With these information, at each layer, the theoretical irradiance at the $435 \pm 20$ and $655 \pm 20 \mathrm{~nm}$ wavelength bands is calculated with Planck's theory and a wavelength-dependent soot absorption function, $E(m)$, as expressed in ref. [19] (for each layer an identical $E(m)$ is used). The resulting signal is integrated across all the layers. After taking the ratio of signals at two wavelength bands, the line-of-sight averaged temperature is calculated to be $1690 \mathrm{~K}$ by two-color ratio pyrometry using a lookup table approach [20]. This temperature was used as the reference temperature to calibrate the experimental setup and shown in Fig. 2 with the dashed black line. Further reducing the layer thickness in this analysis does not provide significant change in the results. 


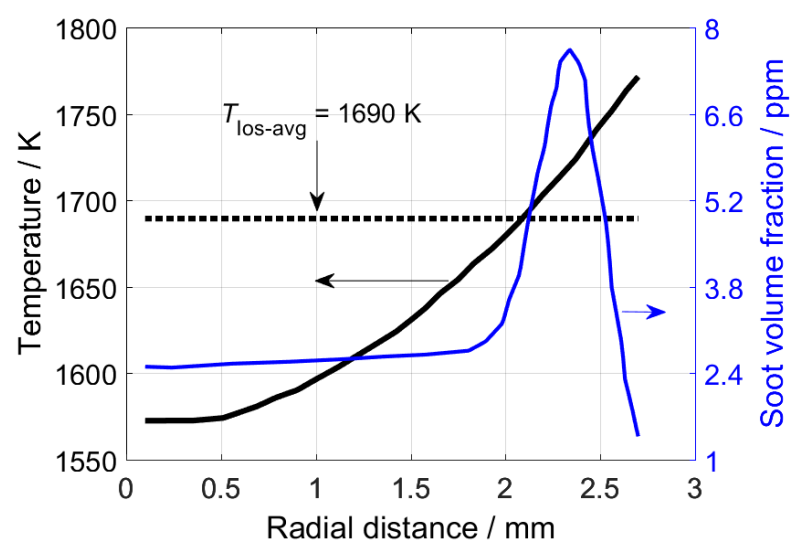

Fig. 2 Line-of-sight averaged flame temperature, $T_{\text {los-avg, }}$ calculated with the temperature and soot volume fraction distribution for the calibration of photomultipliers. Temperature and soot volume fraction distributions at $5 \mathrm{~cm}$ above the burner are imported from the literature.

The soot at the edge is more mature than the soot at the inner layers, and more mature soot is known to have a higher $E(m)$. To identify the related uncertainty, the dependence of $E(m)$ was calculated with another approach [12] that incorporates the effects of wavelength, temperature and soot maturity. The soot maturity is defined in terms of annealed mass fraction (see section 3 ) and set to 0.2 at the flame axis and 0.8 at the outermost flame edge. These annealed mass fractions are believed to be reasonable estimations for each of the locations. It indicates that $20 \%$ of the soot shows graphitic behavior at the flame center, whereas the relative population of graphitized soot is four times greater at the edge. Taking the radial temperature distribution also into account (see Fig. 2), the change of $E(m)$ from center to edge at the steady state is calculated as $-5.1 \%$ and $9.5 \%$ for the 435 and $655 \mathrm{~nm}$ wavelength, respectively. Based on these variations, the line-of-sight integrated theoretical emission is recalculated (each layer has a unique $E(m)$, and the change of $E(m)$ from center to edge is linearly interpolated across the layers) for the two color bands. The ratio of two theoretical signals is again interpreted with the lookup table based on the fixed $E(m)$ (only wavelength dependent) as expressed above, and the line-of-sight averaged temperature is calculated to be only $1.5 \mathrm{~K}$ smaller than the previously calculated value. This highly small deviation shows that the expression shown in ref. [19] for $E(m)$ is a reasonable estimation for the soot at the flame temperature and over the visible spectra. Hence, the soot-maturity related bias in two-color pyrometry is negligible for the flame temperature measurements (effects of $E(m)$ on temperature measurements are further discussed in section 4).

Energy of each laser's pulse was measured by an energy-meter (Coherent FieldMaxll-TOP) which measure the area integrated energy. Inhomogeneities, specifically hotter spots, in the beams' spatial profiles may heat the soot 
locally more than area-averaged value, and eventually bias the pyrometric temperature detection to higher values. To verify the similarity of two lasers, the heat-up temperature at peak LII signal was observed for individual laser pulses (one laser at a time) at various fluences. The measured heat-up temperature during the pulse of first laser at different laser energies were compared to the reference heat-up temperatures achieved by the secondary laser without the presence of first laser. A map for the secondary laser energy settings (number of beam splitters, N.D. filters and the Q-switch delay) was created so that the heat-up temperatures at the designated fluences are identical to those from the reference first laser. In this calibration, the deviation from a conventional energy-meter based mapping was always less than $10 \%$. The effects on laser heating related to possible variation of the tempospatial profiles at different laser energies were marginal and they were corrected for when necessary via fineadjustment of pulse energy.

\section{Modeling}

Particle-size measurements with time-resolved LII are based on the fact that, after the laser pulse, smaller particles cool down faster than larger ones due to their larger surface-to-volume ratio [1]. In a quantitative particle-sizing approach, multiple numerical LII models have been developed and a comparison of these models is shown in [21]. For this present work, a new LII simulation tool is developed. The software incorporates well-established submodels to simulate particle heating through absorption of laser light and thermal annealing, as well as cooling through sublimation, radiation, thermionic emission, and conduction. Particle mass loss is accounted for through the sublimation term. Particle density, specific heat, and the thermal accommodation coefficient (TAC) utilized in the model are temperature dependent (or can be set to constant values). In addition, thermodynamic and optical properties are phase dependent, meaning that they are changing due to the annealing effects.

Laser absorption is modeled as the product of the normalized laser intensity profile weighted by the laser fluence and the absorption cross-section as detailed in [11]. In this model the total absorbed energy is computed as diameter, wavelength, and annealed mass fraction dependent. Heat loss through radiation is modeled by the Planck equation as described in [22] with an emissivity correction accounting for annealing [11]. For heat conduction, the Fuchs approach [23] is chosen. Sublimation of $C_{1}-C_{5}$ carbon clusters is included in the model as used in the work of Hofmann et al. [22] and is described in [21]. Thermionic emission is modeled according to ref. [24].

Thermal annealing is modeled following the work of Michelsen and is described in [11]. According to this model, annealing occurs at temperatures high enough to allow for the migration of interstitial carbon atoms in a process known as Frenkel defect annihilation. At even higher temperatures, clustering of lattice vacancies is modeled as 
Schottky defect annihilation. The annealing rate is computed as the rate of defect formation minus the rates of defect annihilation given in Eq. (1) and (2).

$\frac{d N_{d}}{d t}=X_{a n n} \frac{N_{p}}{2} k_{\text {diss }}-k_{\text {imig }} N_{d}-k_{v m i g} N_{d}$

$X_{a n n}=1-\frac{N_{d}}{X_{d} N_{p}}$

In these equations $N_{d}$ is the number of defects, $N_{\mathrm{p}}$ is the number of atoms in the particle, $X_{\mathrm{d}}$ is the initial defect density of the particle, $X_{\mathrm{ann}}$ is the fraction of the particle that has undergone annealing and represents the fine structure order and maturity of the particle $\left(X_{\text {ann }}\right.$ increases as $\mathrm{H} / \mathrm{C}$ decreases; when $X_{\text {ann }}$ reaches one, $\mathrm{H} / \mathrm{C}$ is zero [12]), and $k_{\text {diss, }} k_{\text {imig, }} k_{\mathrm{vmig}}$ are the rate constant for pyrolysis of the annealed particle, for interstitial migration, and for vacancy migration, respectively. These rate constants in Eq. (1) are calculated with Arrhenius equations and they increase as the particle temperature increases. At moderate temperatures, these rate constants increase exponentially with temperature and level off as they approach to their limit. Parameters to calculate these rate constants shown in [11] are determined through a combination of theoretical analysis as well as best-fit analysis of experimental data.

In the newly developed LII simulation tool, the energy- and mass-balance equations are solved numerically with a fourth-order Runge-Kutta algorithm with the above mentioned sub-models. The time-dependent particle temperature is turned into LII signal intensity using Planck's law after emissivity correction and integrated over a user-defined wavelength band. Experimental LII data can also be fitted. With the chosen initial parameters, the particle diameter is determined by a Levenberg-Marquardt non-linear least-squares fitting algorithm. To include the effects of bridging and aggregation on the conduction model, the shielding factor is calculated according to the work of Johnsson et al. [25]. In this aggregation sub-model, a monodisperse aggregate size is used and the number of primary particles, $N_{\mathrm{p}}$, is set to 100 which is imported from an existing TEM data set [14]. The fractal prefactor $k_{\mathrm{f}}$, fractal dimension $D_{\mathrm{f}}$ and the overlap parameter $C_{\mathrm{ov}}$ are imported from [25]. Thermophysical particle properties, as given in [11], are temperature dependent. Soot absorption function, $E(m)$, is wavelength dependent and calculated as shown in [19]. To be consistent with the aggregation model, the thermal accommodation coefficient, TAC is set to 0.37 , as evaluated by Snelling et al. [19] for a laminar ethylene co-flow diffusion flame. For the purposes of this study, polydispersion modelling was switched off. Relevant initial conditions used in the theoretical analysis are given in Tab. 1. The particle diameter is decided according to ref. [14] and the bath-gas temperature, $T_{\text {bathgas, }}$ is interpolated for the axial and radial position given in section 2 according to the temperature map given in [15]. 


\begin{tabular}{|l|c|}
\hline Particle diameter & $30 \mathrm{~nm}$ \\
\hline$T_{\text {bathgas }}$ & $1600 \mathrm{~K}$ \\
\hline Laser fluence & $0-0.18 \mathrm{~J} / \mathrm{cm}^{2}$ \\
\hline Laser pulse duration & Gaussian: $7 \mathrm{~ns}$ FWHM \\
\hline$E(m)$ & wavelength dependent \\
\hline TAC, $N_{\mathrm{p}}, k_{\mathrm{f}}, D_{\mathrm{f}}, C_{\mathrm{ov}}$ & $0.37,100,2.3,1.8,0.125$ \\
\hline Soot density & $1860 \mathrm{~kg} / \mathrm{m}^{3}$ \\
\hline
\end{tabular}

Tab. 1 Initial conditions for LII model.

In an effort to gain insight into the behavior of the LII model, simulations were carried out which were comprised of two laser pulses (see Tab. 1 for the other relevant conditions). In a two-pulse LII system, where soot is preheated with the first pulse, the LII signal decay after the consecutive second pulse must be a sensitive measure of the particle annealing. To provide an isolated measure of the changes induced solely by pulse one (named as pump pulse hereafter), the second pulse (named as probe pulse hereafter) is set to a constant low fluence of $0.07 \mathrm{~J} / \mathrm{cm}^{2}$ to minimize its perturbation on the soot, and the fluence of the pump pulse is varied from 0 to $0.18 \mathrm{~J} / \mathrm{cm}^{2}$. The entire duration of simulation is set to $15 \mu \mathrm{s}$ and the time separation between the two laser pulses was held constant at $11 \mu \mathrm{s}$, a value sufficient to allow the soot heated by the pump pulse to cool to the local flame temperature (bath-gas heating is neglected in these simulations). During this $11 \mu$ s delay between pulses, a soot particle in the standard flame at $5 \mathrm{~cm}$ above the Santoro burner travels less than $22 \mu \mathrm{m}$. This distance is sufficiently short to assume that the probe volume is not flushed with fresh gas, and these two consecutive laser pulses heat the very same soot mass.

To assess the performance of the annealing subroutine, simulations were performed with two different conditions. In the first scenario, the annealing model was switched off. Thus, the pump pulse has no effects on the internal structure of the soot particle and the heating of soot during the probe pulse. In the second scenario, the annealing routine was turned on. Based on values measured for graphite, the emissivity and absorption cross section of carbon decreases as particles anneal (Fig. 4 in [11]). Thus, if the particle anneals during the laser pulse, the integrated value of the laser-heating term and cooling term via radiation are reduced. The resulting heat-up temperatures of soot during the probe pulse were compared for the two scenarios, namely with and without annealing sub-model. The heat-up temperature of the annealed soot is $400 \mathrm{~K}$ less compared to unannealed soot. As mentioned earlier, the Michelsen group [12] recently reported a modified expression for soot absorption function which predicts an enhancement of light absorption rate after the graphitization. 
These simulations also showed that, with the parameter set given in [11], the annealing also occurs rapidly at very low temperatures. With an initial defect density, $X_{\mathrm{d}}$, of 0.01 , the complete graphitization $\left(X_{\mathrm{ann}}>95 \%\right)$ of a soot particle at the flame temperature of $1600 \mathrm{~K}$ (i.e., without laser heating) takes less than $15 \mu$ s. Specifically for the current simulations, $90 \%$ of the defects in the soot particle (their initial population is $1 \%$ of the total number of atoms) are annihilated at the time of probe pulse ( $>11 \mu \mathrm{s}$ after the start of simulation). The temporal profile of the particles' annealed mass fraction is plotted for increasing pump pulse fluences and no laser heating in Fig. 3. As mentioned above, the annealing model leads a complete graphitization of the soot at the flame temperature independent of the pump laser fluence. With the increasing pump pulse fluence, and hence the heat-up temperature, however, the conversion rate increases and graphitization is completed at much shorter durations. In this modeling work, fluences above $0.18 \mathrm{~J} / \mathrm{cm}^{2}$ were not analyzed. Extending the fluence range to higher values, however, does not change the heat-up temperature during the probe pulse as the sublimation model has no effects on the internal structure of the soot; the peak LII signal reduces due to evaporated solid phase soot. The acceleration of graphitization at $11 \mu \mathrm{s}$ is due to the secondary heating with the probe pulse.

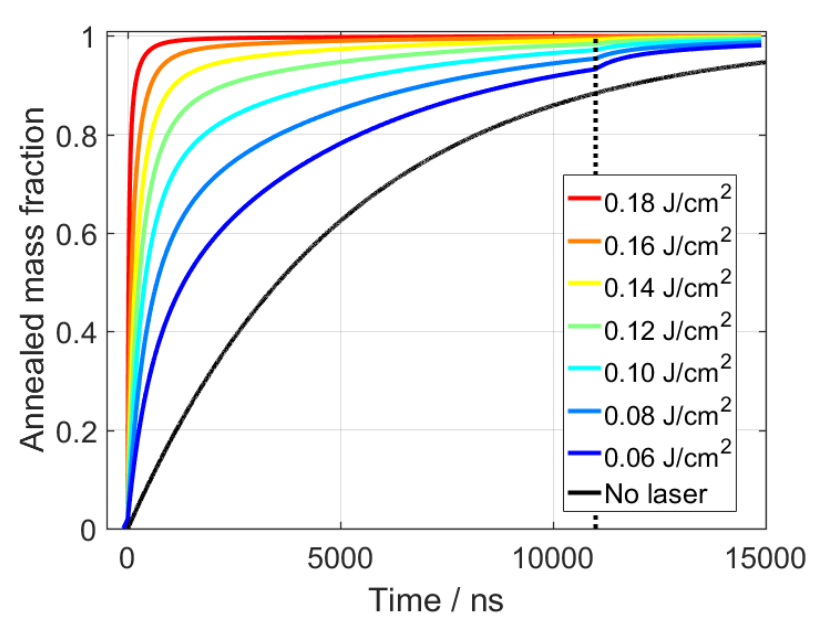

Fig. 3 Temporal evolution of annealed mass fraction. Dotted vertical line is the timing of probe pulse.

Under standard operating conditions of the Santoro burner, a soot particle completes its in-flame journey from inception zone to oxidation over much longer time scales. However, none of the TEM analysis reported significant annealing of in-flame soot sampled at the center without the presence of external laser heating. In ref. [12] it is reported that soot primary particles commence to anneal at temperatures above $\sim 2500 \mathrm{~K}$ into more ordered structures. Therefore, it can be concluded that the annealing model with the parameter set (rate constants and initial defect density) given in ref. [11] predicts a too rapid graphitization of soot at low temperatures. It should 
be also noted that the annealing of cold soot at temperatures below $1600 \mathrm{~K}$ was previously observed with TEM analysis; however, the duration of the heat treatment was from 7 [26] to 18 [27] hours.

\section{Results and discussion}

\subsection{Two-color pyrometry and effects of annealing (experiments)}

The pump-probe pulse LII experiments were performed as introduced in the modeling efforts in section 3 . The probe pulse was set to a constant fluence of $0.07 \mathrm{~J} / \mathrm{cm}^{2}$, and the pump pulse fluence was varied from 0 to 0.33 $\mathrm{J} / \mathrm{cm}^{2}$ over 22 increments. The time separation between the two laser pulses was held constant at $11 \mu \mathrm{s}$. The signal intensity was acquired for the entire two-pulse LII process (15 $\mu \mathrm{s})$. It is verified that the heated soot cools back to the flame temperature at all pump laser fluences within the given separation time (a residual temperature increase of $\sim 100 \mathrm{~K}$ remains due to the bath gas heating). The resulting heat-up temperatures are shown in Fig. $4 \mathrm{a}$ for both the pump and the probe pulses. The signal collection setup and the gain settings of the PMTs were set to maximize the resolution of the measurements at the probe pulse. Therefore pump pulse heat-up temperatures above a fluence of $0.12 \mathrm{~J} / \mathrm{cm}^{2}$ could not be measured due to saturation of photomultipliers, i.e. loss of linearresponse of photomultipliers. To ensure repeatability of temperature measurements during the two-pulse LII experiments, the acquired signals were averaged over 500 single measurements. The standard deviation of the heat-up temperature at the probe-pulse over 500 single measurements was measured to be $31 \mathrm{~K}$ and shown with error-bars in the Fig. 4a on the probe pulse measurements. This measured precision span is associated to shot noise in the detectors, flame fluctuations and laser shot-to-shot variations. The accuracy of the temperature measurement is weakly dependent on the assumed values of $E(m)$ at the detection color bands [19], as also shown in section 2 for the spectral calibration of detectors. Therefore effects of annealing-related changes of the $E(m)$ on temperature measurements are insignificant. The change of the absolute value of $E(m)$, however, is critical for the absorption of energy. 
(a)

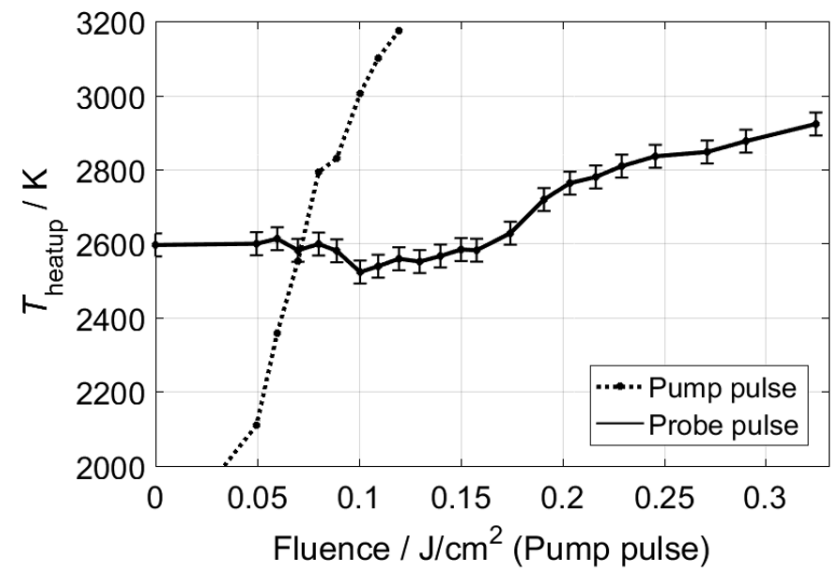

(b)

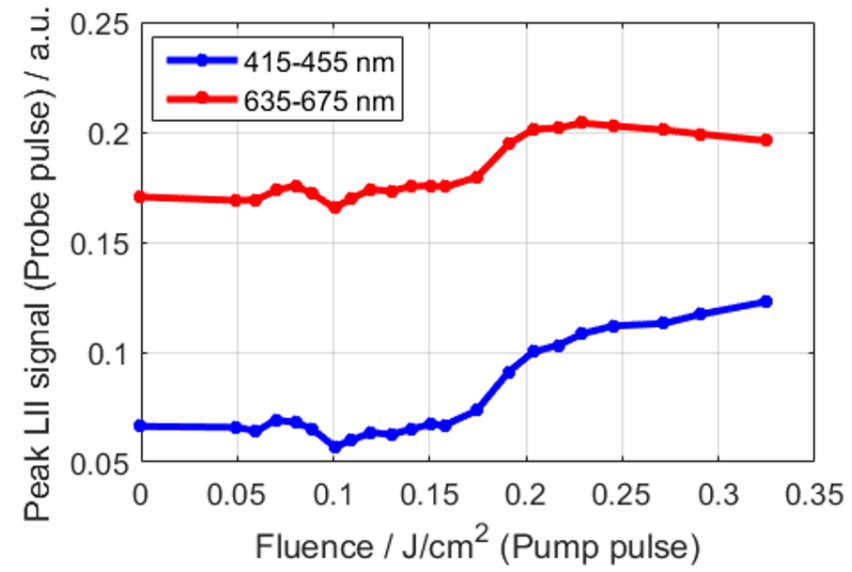

Fig. 4 a Measured heat-up temperature of soot at the pump and probe pulse at different pump fluences. b Peak LII signals from the two channels at the probe pulse.

The measurements show that any pre-heating of in-flame soot below a fluence of $0.17 \mathrm{~J} / \mathrm{cm}^{2}$ (the simulations indicates that this corresponds to a temperature of below $4000 \mathrm{~K}$ with an $E(m)$ defined by Snelling et al. [19]) does not lead to a major systematic change in the heat-up temperature of in-flame soot, hence its absorption characteristics, at the time of the probe pulse. The behavior of the heat-up temperature for the cases of preheating from 0.05 to $0.17 \mathrm{~J} / \mathrm{cm} 2$ is disputable as there is not an obvious trend in the curve. For a conclusive analysis at low fluence, a measurement system with lower precision uncertainty is necessary. At $0.07 \mathrm{~J} / \mathrm{cm}^{2}$, where pump and probe pulse fluences are equal, the readout for heat-up temperatures are also almost equal. This indicates that the soot properties are unchanged between these two equal pulses (at this fluence residual bathgas heating after the pump pulse is calculated as $\sim 30 \mathrm{~K}$ with the LII modeling for a soot volume fraction of $3 \mathrm{ppm})$. This outcome is partially in contradiction to the work of Vander Wal et al. [9] which reported changes of soot properties via heating of pump pulses with similar fluences $\left(<0.1 \mathrm{~J} / \mathrm{cm}^{2}\right)$. However, conclusions in that study were extracted from the peak LII signal intensity measured at a single color and there was only one low fluence measurement.

In the present work, the heat-up temperature at the probe pulse systematically increases at pump-pulse fluences above $0.17 \mathrm{~J} / \mathrm{cm}^{2}$, which can be associated with the increased emissivity and absorption of soot due to the thermal annealing with the pump pulse. Additionally increased residual bath-gas heating causes a higher temperature read-out at the probe pulse. The recent work of Saffaripour et al. [10], based on extinction measurements, confirms this outcome. Vander Wal et al. [9] also observed a positive change trend in the absorption characteristics of soot after graphitization. Such enhancement of these optical characteristics, however, 
contradicts the effects predicted in simulations with the annealing sub-model shown in section 3. Evidently, the temperature (pump-pulse fluence) threshold measured for the activation of soot graphitization is also in contradiction with the simulations.

To understand the inconsistency with the works of Vander Wal et al. [9], the acquired peak LII signals from two color channels are also shown for increasing pump-pulse fluence in Fig. 4b. The peak LII intensity is proportional to a combination of soot volume fraction, effective primary particle size, emissivity, and the temperature. The latter one is dependent on heat absorption, hence graphitization. Therefore only by looking at the peak LII signal, it is difficult to isolate the effects of pre-heating on the optical characteristics of soot and effects of sublimation on the particle size (see section 4.2). Continuously increasing signal levels at higher fluences are due to the increasing soot surface temperature. The loss of solid material due to sublimation at increasing laser fluences, on the other hand, causes a reduction of the LII signal. Therefore increased temperature and sublimation have competing effects on the LII signal intensity. At the $415-455 \mathrm{~nm}$ wavelength band (blue curve), the trend of the signal is very similar to the heat-up temperature at the probe pulse. The LII signal is unchanged at low fluence regimes of the pump pulse (neither annealing nor sublimation are considerably effective), and then continuously increases at higher fluences with the temperature. This shows that the constructive effects of temperature increase (related to graphitization) dominate over the destructive effects of sublimation on the LII signal. At 655 $\mathrm{nm}$ wavelength, however, the LII intensity starts to decrease slightly beyond $0.25 \mathrm{~J} / \mathrm{cm}^{2}$ after the initial increase onset. This discrepancy at different colors can be explained in the following way: Assuming a linear relation between $E(m)$ and wavelength [19], the integrated Planck irradiation at $415-455 \mathrm{~nm}$ wavelength band increases more than four times from 2600 to $2923 \mathrm{~K}$ (heat-up temperatures at probe pulse after heating with pump-pulse fluence of 0.17 and $0.33 \mathrm{~J} / \mathrm{cm}^{2}$, respectively). It should be noted that the increase of the LII signal, shown in Fig. $4 \mathrm{~b}$, is less than a factor of two and this is because of the destructive effects of sublimation on the LII signal. At 635 - $675 \mathrm{~nm}$ wavelength band, however, the theoretical increase of Planck irradiation is only 2.55 times as a result of Wien's displacement law. When this relatively weaker signal enhancement is coupled with lowering effects of soot sublimation, the net balance is negative above $0.25 \mathrm{~J} / \mathrm{cm}^{2}$. With the two-color pyrometry approach (in which both temperature and particle size information available) as employed in this current study, analyzing the effects of rapid heating on soot particles and LII signal can be performed with a higher accuracy than measurements based on single color as shown in [9].

\subsection{Particle sizing and effects of sublimation}

To see the sublimation effects of rapid pre-heating on soot, particle sizes were determined from the LII-signal decay curves acquired after the probe pulse. In the curve fitting, soot absorption part was omitted, and fitting was 
performed with the decay signal acquired at $655 \pm 20 \mathrm{~nm}$ wavelength, and the measured heat-up temperature, shown in Fig. 4a, was used to set the initial temperature. The acquired data at this color band has a better signalto-noise ratio than the blue channel and therefore it is selected for the particle sizing. Alternatively the fitting could be performed with the time-resolved temperature information. However, no additional benefit in accuracy was observed (contrarily, due to the superimposed noise from two channels, a worse signal-to-noise ratio was observed at lower temperature towards the end of LII signal). Other relevant initial conditions for the model are given in Tab. 1. The annealing sub-model was turned off for all the calculations as the current model cannot predict the changes in soot accurately, such as graphitization rate and $E(m)$ (see section 3 and 4.1). For all the pump-pulse fluences, identical soot properties (emissivity and density) were used. Curves were smoothened with a Butterworth filter and normalized with their respective peak signal (smoothing filter does not affect the performance of the curve-fitting algorithm). Background signal was subtracted. Two typical measured LII data (pump laser fluence 0.05 and $0.33 \mathrm{~J} / \mathrm{cm}^{2}$ ) and the respective best-fit for these curves are shown in Fig. 5. Only the first $2000 \mathrm{~ns}$ after the probe pulse of LII decay curves are used. The goodness of the fitting for all pump-pulse fluences was measured by the coefficient of determination $R^{2}$, and also shown in Fig. 5 with the black line (upper and right axis). 


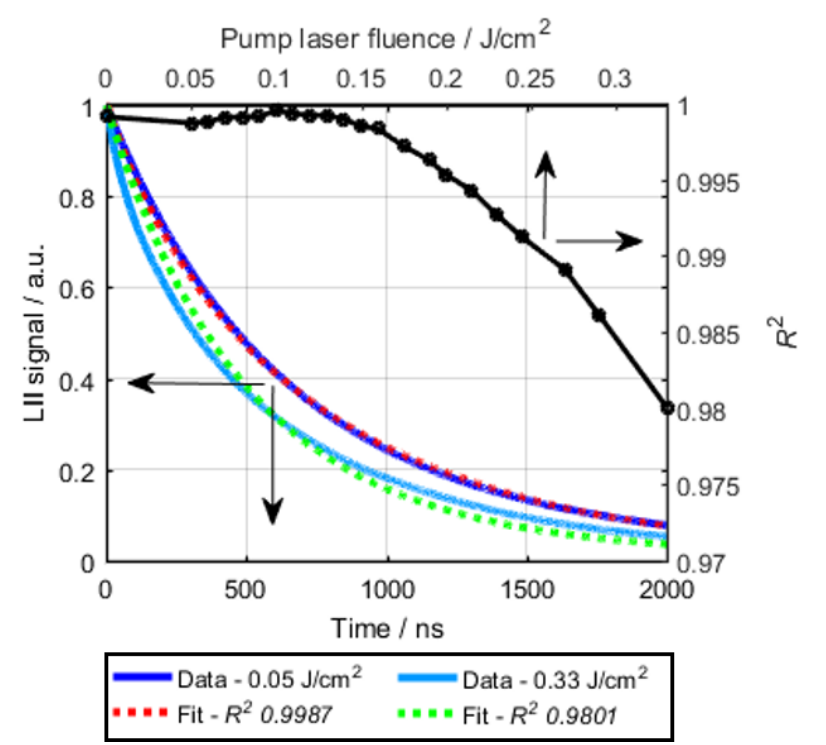

Fig. 5 Typical measured TiRe-LII signal traces at $655 \pm 20 \mathrm{~nm}$ wavelength after the probe pulse and the respective best-fit curves, for pump fluence of 0.05 and $0.33 \mathrm{~J} / \mathrm{cm}^{2}$. The goodness of the fitting is shown with $R^{2}$ (upper $x$-axis and right $y$-axis) for all 22 different pump fluences (black line).

For increasing fluences, the goodness of the fitting worsens. Among the governing cooling mechanisms mentioned in section 3, the radiation has a minor contribution to the overall energy exchange (for the conditions given in Tab. 1, it is responsible only for less than $2 \%$ of the total heat loss). During the cooling process, $E(m)$ is only effective on the radiation mechanism (as the heat-up temperature is imported from the two-color pyrometry measurements, the absorption does not need to be considered). To assess the sensitivity of the evaluated $d_{\mathrm{p}}$ to the value of $E(m)$, curve-fittings shown in Fig. 6 were performed with fixed $E(m)=0.2$ and $E(m)=0.4$. For each fitting, the difference of the evaluated particle diameter is not greater than $0.8 \mathrm{~nm}$ between the two values of $E(m)$. Therefore effects of $E(m)$ on the curve-fitting and the predicted particle size can be neglected. The density of soot, on the other hand, can be significantly effective on particle sizing as the internal energy is directly dependent to this property. A $10 \%$ increase in the assumed soot density causes roughly a $10 \%$ reduction in the predicted particle size. Similarly for a $10 \%$ smaller soot density input in the model, a $10 \%$ larger particle diameter is predicted. The dropping $R^{2}$ for increasing pre-heating can be associated with the changing thermophysical properties of soot, specifically density, with annealing which was omitted in the LII model.

Twenty-two recorded LII signal with different pre-heating conditions from 0 to $0.33 \mathrm{~J} / \mathrm{cm}^{2}$ are shown in Fig. 6 in color scale. With increasing pump laser fluence, the decay rate of the signal increases, and curves become steeper (the decay signals for the pre-heating conditions of pump-pulse fluence below $0.1 \mathrm{~J} / \mathrm{cm}^{2}$ are almost identical and 
overlapping). The predicted soot particle sizes for all pump laser conditions are also shown in Fig. 6 (black line upper and right axis). Associated uncertainties in LII particle-sizing due to false initial and boundary conditions are previously shown in [14] for a similar LII modeling tool. The predicted particle size with the current LII simulator is a monodisperse equivalent particle size of the poly-disperse soot ensemble. The particle-size measurements at the single pulse case (pump pulse is turned off) shows good agreement with the TEM measurements shown in [14]. The first reduction of the particle diameter, via sublimation, is observed at laser fluences as low as $0.1 \mathrm{~J} / \mathrm{cm}^{2}$, which corresponds to a heat-up temperature of $3000 \mathrm{~K}$ at the pump pulse $(E(m)$ as defined in [19] and shown in Fig. 4a). Although this sublimation threshold is lower than the previously published model-based estimations [28] and experimental data based on graphite cited in [11], it is in very good agreement with the recently measured data [10] for in-flame soot in a similar flame. With increasing pre-heating, the particle size at the probe pulse gradually decreases. At $0.33 \mathrm{~J} / \mathrm{cm}^{2}$, the particle loses $55 \%$ of its initial mass. It should be noted that at higher fluence, the decay course of the simulated curves starts to differ from the acquired signal, and goodness of the fitting decreases (see Fig. 5). This means that the predicted particle sizes for higher pump-pulse fluence scenarios are less reliable. Nevertheless, as can be seen from the reducing acquired signal lifetimes at higher fluences, the shrinkage trend in the particle size is correct. 


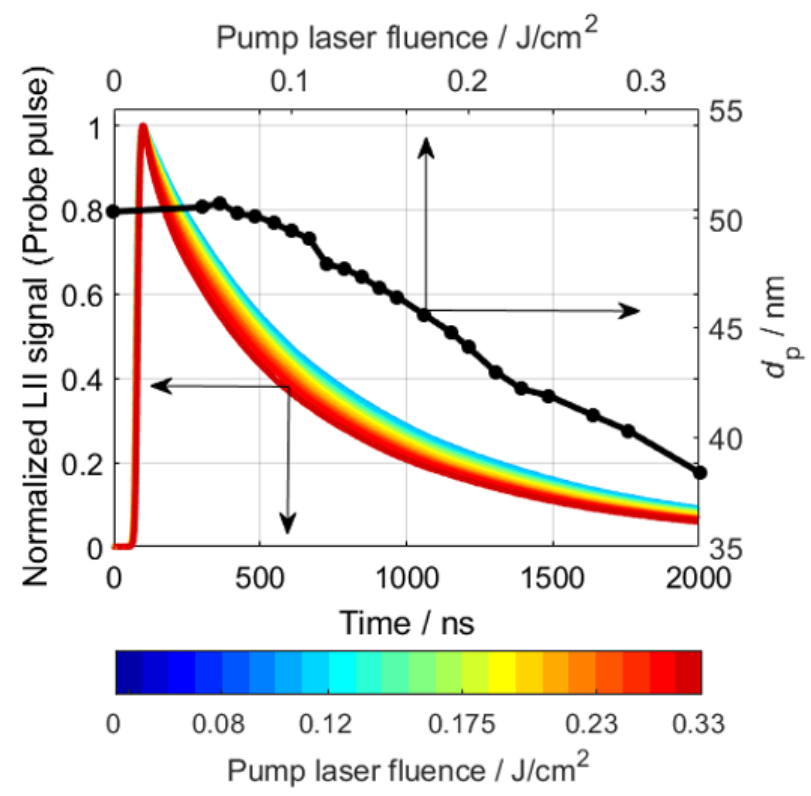

Fig. 6 Measured TiRe-LII signal traces at $655 \pm 20 \mathrm{~nm}$ wavelength under 22 pre-heating conditions (normalized to peak signal and plotted in color scale as shown in the horizontal colorbar). Determined particle diameter (upper $x$-axis and right $y$-axis) is shown with the thick black line.

\section{Conclusions}

Overall effects of annealing and sublimation processes on optical properties of in-flame soot and LII model are investigated. Optical measurements are performed with a two-pulse LII system, where soot is preheated with the first pump pulse, and the time resolved LII signal after the consecutive probe pulse is analyzed. The LII signals are acquired at two different colors and the temperature of the soot is determined by means of pyrometry. A newly developed LII simulation software is used to assess the accountability of rapid heating processes in LII modeling.

The annealing model shown in this work appears to contradict the experimental outcome presented. The model assumes that soot commences to anneal at temperatures as low as flame temperature whereas experiments showed that annealing starts above $\sim 4000 \mathrm{~K}$. The model suggests that annealing leads to a reduction in the absorption and emissivity characteristics of soot whereas experiments indicated a noticeable enhancement of such optical properties once soot is annealed, in agreement to similar previous experimental works. For particle sizing of in-flame soot with low laser fluences $\left(<\sim 0.15 \mathrm{~J} / \mathrm{cm}^{2}\right)$, the annealing mechanism can be overlooked in LII modeling.

The two-color two-pulse LII results showed differences with some previous two-pulse LII works in which the conclusions were extracted from peak LII signal intensity and decay curves at single color. With a detailed analysis 
of LII signal at different wavelengths and the particle temperature, the possible reason of discrepancies is explained. For assessment of annealing effects on soot, the necessity of particle temperature information during the two-pulse LII process is shown. In agreement with the recent findings, LII measurements showed that the sublimation of in-flame particle starts at a laser fluence of $\sim 0.1 \mathrm{~J} / \mathrm{cm}^{2}$, which corresponds to a heat-up temperature of $3000 \mathrm{~K}$. At a laser fluence of $0.33 \mathrm{~J} / \mathrm{cm}^{2}$, the mass loss of the particle due to sublimation is measured as $55 \%$ of its initial mass.

\section{Acknowledgements}

The research reported in this publication was supported by funding from King Abdullah University of Science and Technology (KAUST). 


\section{References}

[1] Melton, L. A., Appl. Opt. 23 (1984) 2201-2208.

[2] Schulz, C., Kock, B. F., Hofmann, M., Michelsen, H. A., Will, S., Bougie, B., Suntz, R., and Smallwood, G. J., Appl. Phys. B 83 (2006) 333-354.

[3] Vander Wal, R. L., Choi, M. Y., and Lee, K. O., Combust. Flame 102 (1995) 200-204.

[4] Vander Wal, R. L., and Jensen, K. A., Appl. Opt. 37 (1998) 1607-1616.

[5] Vander Wal, R. L., and Choi, M. Y., Carbon 37 (1999) 231-239.

[6] De luliis, S., Cignoli, F., Maffi, S., and Zizak, G., Appl. Phys. B 104 (2011) 321-330.

[7] Michelsen, H. A., Tivanski, A. V., Gilles, M. K., van Poppel, L. H., Dansson, M. A., and Buseck, P. R., Appl. Opt. 46 (2007) 959-977.

[8] Bambha, R. P., Dansson, M. A., Schrader, P. E., and Michelsen, H. A., Appl. Phys. B 112 (2013) 343-358.

[9] Vander Wal, R. L., Ticich, T. M., and Stephens, A. B., Appl. Phys. B 67 (1998) 115-123.

[10] Saffaripour, M., Geigle, K.-P., Snelling, D. R., Smallwood, G. J., and Thomson, K. A., Appl. Phys. B 119 (2015) 621-642.

[11] Michelsen, H. A., J. Chem. Phys. 118 (2003) 7012-7045.

[12] López-Yglesias, X., Schrader, P. E., and Michelsen, H. A., J. Aerosol Sci. 75 (2014) 43-64.

[13] Santoro, R. J., Semerjian, H. G., and Dobbins, R. A., Combust. Flame 51 (1983) 203-218.

[14] Cenker, E., Bruneaux, G., Dreier, T., and Schulz, C., Appl. Phys. B 118 (2015) 169-183.

[15] Santoro, R. J., Yeh, T. T., Horvath, J. J., and Semerjian, H. G., Combust. Sci. Technol. 53 (1987) 89-115.

[16] Liu, F., Snelling, D. R., Thomson, K. A., and Smallwood, G. J., Appl. Phys. B 96 (2009) 623-636.

[17] Cenker, E., Kondo, K., Bruneaux, G., Dreier, T., Aizawa, T., and Schulz, C., Appl. Phys. B 119 (2015) 765-776.

[18] Connelly, B. C., Quantitative characterization of steady and time-varying, sooting, laminar diffusion flames using optical techniques (Doctoral dissertation), PhD thesis, Yale University, (2009).

[19] Snelling, D. R., Liu, F., Smallwood, G. J., and Gülder, Ö. L., Combust. Flame 136 (2004) 180-190.

[20] Kuhn, P. B., Ma, B., Connelly, B. C., Smooke, M. D., and Long, M. B., Proc. Combust. Inst. 33 (2011) 743-750.

[21] Michelsen, H. A., Liu, F., Kock, B. F., Bladh, H., Boiarciuc, A., Charwath, M., Dreier, T., Hadef, R., Hofmann, M., Reimann, J., et al., Appl. Phys. B 87 (2007) 503-521.

[22] Hofmann, M., Kock, B. F., Dreier, T., Jander, H., and Schulz, C., Appl. Phys. B 90 (2007) 629-639. 
[23] Filippov, A. V., and Rosner, D. E., Int. J. Heat Mass Transf. 43 (2000) 127-138.

[24] Mitrani, J. M., Shneider, M. N., Stratton, B. C., and Raitses, Y., Appl. Phys. Lett. 108 (2016) 54101.

[25] Johnsson, J., Bladh, H., Olofsson, N.-E., and Bengtsson, P.-E., Appl. Phys. B 112 (2013) 321-332.

[26] Dunne, L. J., Nolan, P. F., Munn, J., Terrones, M., Jones, T., Kathirgamanathan, P., Fernandez, J., and Hudson, A. D., J. Physics-Condensed Matter 9 (1997) 10661-10673.

[27] Bacsa, W. S., de Heer, W. A., Ugarte, D., and Châtelain, A., Chem. Phys. Lett. 211 (1993) 346-352.

[28] Liu, F., Stagg, B. J., Snelling, D. R., and Smallwood, G. J., Int. J. Heat Mass Transf. 49 (2006) 777-788. 